\title{
AFFINE SPHERES AND KÄHLER-EINSTEIN METRICS
}

\author{
John C. LOFTIN
}

\section{Introduction}

In this note, we introduce a straightforward correspondence between some natural affine Kähler metrics on convex cones and natural metrics on certain hypersurfaces asymptotic to the boundary of these cones. Recall an affine Kähler metric is a Riemannian metric locally given by the Hessian of a potential function $\phi$, i.e. $g_{i j}=\frac{\partial^{2} \phi}{\partial x^{i} \partial x^{j}}$. Note that this metric is well defined only up to affine coordinate changes. Affine Kähler metrics are sometimes called Hessian metrics. See e.g. $[4,12]$.

The centroaffine second fundamental form provides a Riemannian metric on a hypersurface $H \subset \mathbb{R}^{n+1}$, if $H$ is the radial graph of a function $-\frac{1}{u}$, with $u$ negative and convex. The formula for this metric is $-\frac{1}{u} \frac{\partial^{2} u}{\partial t^{2} \partial t^{j}}$, and if $u$ transforms as section of a certain line bundle, this metric makes sense under projective coordinate changes. See e.g. [10].

Form a cone

$$
\mathcal{C}=\bigcup_{s>0} s H
$$

of homothetic copies of $H$. Then consider the affine Kähler metric given by the potential function $\log \sigma$, where $\sigma$ is homogeneous of degree $-p$ and constant on $H$. In Section 3 below we show that (up to a constant) this affine Kähler metric naturally splits along the foliation (1) into an orthogonal sum of a radial metric $\frac{d s^{2}}{s^{2}}$ and the centroaffine metric on $H$. The Cheng-Yau metric and the Koszul-Vinberg metric are both affine Kähler metrics of this form with $p=n+1$.

All these notions are invariant under linear isomorphisms of the cone $\mathcal{C}$, and thus make sense on affine flat manifolds. If the centroaffine metric is complete, then we see that the corresponding affine Kähler metric is complete, and then a result of Shima-Yagi [13] shows that the universal cover of an $\mathbb{R} \mathbb{P}^{n}$ manifold with complete metric $-\frac{1}{u} u_{i j}$ must be a bounded convex domain. This extends the main result of $[10]$ to $\mathbb{R P}^{n}$ manifolds with complete centroaffine metrics. Moreover, we may avoid the hard analysis of [10]. This gives some hope to approach structure theorems on $\mathbb{R} \mathbb{P}^{n}$ manifolds which admit centroaffine second fundamental forms which are not strictly positive definite.

Received February 11, 2002. 
In section 5, we show that this natural correspondence gives a link between two natural geometric Monge-Ampère equations, the affine sphere equation

$$
\operatorname{det}\left(\frac{\partial^{2} u}{\partial t^{i} \partial t^{j}}\right)=\left(-\frac{L}{u}\right)^{n+2},
$$

and Cheng-Yau's equation

$$
\phi=c \log \operatorname{det}\left(\frac{\partial^{2} \phi}{\partial x^{i} \partial x^{j}}\right),
$$

where $c$ and $L$ are positive constants and $u$ and $\phi$ are strictly convex. In particular, for the affine sphere given by the radial graph of $-\frac{1}{u}$ for a solution $u$ of (2), the corresponding affine Kähler metric is a Cheng-Yau metric, given by $g_{i j}=\frac{\partial^{2} \log \phi}{\partial x^{i} \partial x^{j}}$ for $\phi$ solving (3) for an appropriate $c$. For appropriate boundary conditions, these two equations were solved by Cheng and Yau in $[4,3]$ respectively. The fundamental results on the geometry of solutions of (2) are found in Calabi [1] and Cheng-Yau [5].

\section{Some invariant affine Kähler metrics}

Consider a convex bounded domain $\Omega$ in $\mathbb{R}^{n}$. Then, by taking the coordinates in $\mathbb{R}^{n}$ to be inhomogeneous projective coordinates, we have the cone over $\Omega$

$$
\mathcal{C}=s\left(t^{1}, \ldots, t^{n}, 1\right), \quad \text { for } \quad s>0, \quad t=\left(t^{1}, \ldots, t^{n}\right) \in \Omega .
$$

Example. Consider the complete affine-invariant metric of the cone $\mathcal{C}$ found by Cheng-Yau [4]. It is given by

$$
g_{i j} d x^{i} d x^{j}=c \frac{\partial^{2} \log V}{\partial x^{i} \partial x^{j}} d x^{i} d x^{j},
$$

where $c$ is a positive constant and $\omega=V d x^{1} \wedge \cdots \wedge d x^{n+1}$ is the volume form of the metric, i.e. $V^{2}=\operatorname{det} g_{i j}$. Note that putting $\phi=\log V$ in (3) gives rise to such metrics. This Cheng-Yau metric is the restriction to $\mathcal{C}$ of the complete Kähler-Einstein metric with negative Ricci curvature on the tube domain $\mathcal{T}=$ $\mathcal{C}+\sqrt{-1} \mathbb{R}^{n+1}$. (There is always such a relationship between an affine Kähler metric and a Kähler metric on a tube domain: simply extend the affine Kähler potential to be constant along the imaginary fibers to get the Kähler potential.) The affine invariance of the metric follows from the biholomorphic invariance of the Kähler-Einstein metric on the tube domain.

Remark. There are three metrics due to Cheng and Yau in this paper: the affine sphere metric on a hyperbolic affine sphere $H$, the Cheng-Yau affine Kähler metric on the cone $\mathcal{C}$, and the Kähler-Einstein metric on the tube domain over $\mathcal{C}$. We only refer to the second as the "Cheng-Yau metric." Of course, as we'll see below in section 5 , these three metrics are all essentially equivalent. 
Our affine Kähler metric (4) is in particular invariant under positive dilations of the cone $\mathcal{C}$, which are generated by the vector field $X=x^{i} \frac{\partial}{\partial x^{i}}$. The equation $\mathcal{L}_{X} \omega=0$ then implies

$$
x^{i} \frac{\partial \log V}{\partial x^{i}}=-(n+1) .
$$

Therefore, putting $\sigma=V$, the Cheng-Yau metric is of the form

$$
\frac{\partial^{2} \log \sigma}{\partial x^{i} \partial x^{j}}, \quad \sigma \text { homogeneous of degree }-(n+1) .
$$

Example. Koszul [8] and Vinberg [14] have constructed another affine Kähler metric on $\mathcal{C}$ which is invariant under linear automorphisms of $\mathcal{C}$. Consider the dual cone $\mathcal{C}^{*}$, which consists of all linear functionals in the dual space to $\mathbb{R}^{n+1}$ which are positive on $\mathcal{C}$. Then define

$$
f(x)=\int_{\mathcal{C}^{*}} e^{-\psi(x)} d \psi
$$

Then it is straightforward to check that $f$ is homogeneous of degree $-(n+1)$ and that $\frac{\partial^{2} \log f}{\partial x^{i} \partial x^{j}}$ is an invariant affine Kähler metric on $\mathcal{C}$.

Remark. Darvishzadeh and Goldman in [6] used this Koszul-Vinberg metric to construct a Weil-Petersson metric on the deformation space $\mathbb{R P}^{2}(S)$ of convex $\mathbb{R P}^{2}$ structures on a closed oriented surface $S$ of genus $g \geq 2$. This metric fits well with the natural symplectic form to form an almost-complex structure on $\mathbb{R P}^{2}(S)$, but it is unknown if this structure is integrable. Their construction can be carried out with any invariant affine Kähler metric of the form (6), and it is possible that for a good choice of such a metric, we may find an integrable complex structure, and therefore a Kähler metric, on $\mathbb{R P}^{2}(S)$.

\section{The splitting theorem}

Consider a metric on $\mathcal{C}$ given by

$$
g_{i j}=c \frac{\partial^{2} \log \sigma}{\partial x^{i} \partial x^{j}}
$$

for $\sigma$ homogenous of degree $-p$ for $c, p$ positive constants. Then we have $X \log \sigma=-p$. Or in other words,

$$
x^{i} \frac{\partial \log \sigma}{\partial x^{i}}=-p
$$

Apply $\frac{\partial}{\partial x^{j}}$ to this equation to get

$$
x^{i} \frac{\partial^{2} \log \sigma}{\partial x^{i} \partial x^{j}}=-\frac{\partial \log \sigma}{\partial x^{j}} \quad \text { for } \quad j=1, \ldots, n .
$$

Consider the family of hypersurfaces $H_{k}=\{\sigma=k\}$ for constants $k$. 
Theorem 1. Under the affine Kähler metric $g$, the vector field $X$ has constant length and is orthogonal to the hypersurface $H_{k}$. Furthermore, the restriction $\left.g\right|_{H_{k}}$ is a equal to a constant $C_{k}$ times the centroaffine metric $h$ along $H_{k}$. Under the foliation $\mathcal{C}=\bigcup_{s>0} s H_{k}$, the affine Kähler metric $g$ splits

$$
(\mathcal{C}, g)=\left(\mathbb{R}^{+}, c p \frac{d s^{2}}{s^{2}}\right) \oplus\left(H_{k}, c p h\right)
$$

Proof. Consider $Y=y^{i} \frac{\partial}{\partial x^{i}}$ a vector tangent to $H_{k}=\{\sigma=k\}$. Then by (8)

$$
g(X, Y)=c \frac{\partial^{2} \log \sigma}{\partial x^{i} \partial x^{j}} x^{i} y^{j}=-c \frac{\partial \log \sigma}{\partial x^{j}} y^{j}=-c Y(\log \sigma)=0 .
$$

Similarly,

$$
\begin{aligned}
g(X, X) & =c \frac{\partial^{2} \log \sigma}{\partial x^{i} \partial x^{j}} x^{i} x^{j}=-c \frac{\partial \log \sigma}{\partial x^{j}} x^{j} \\
& =-c X(\log \sigma)=c p .
\end{aligned}
$$

Now we'll show that the $g$ restricts to a constant multiple of the centroaffine metric on this hypersurface.

Let $D$ be the canonical flat connection on $\mathcal{C} \subset \mathbb{R}^{n+1}$. Then our affine Kähler metric $g$ is given by

$$
g(A, B)=\left(D_{A} d \log \sigma, B\right) c
$$

where $A, B$ are vectors and $(\cdot, \cdot)$ is the pairing between one forms and vectors. Now $X$ is a transverse vector field to $H_{k}$. So at $x \in H_{k}, \mathbb{R}^{n+1}=T_{x}(\mathcal{C})$ splits into $T_{x}\left(H_{k}\right) \oplus\langle X\rangle$. Then we have

$$
\begin{aligned}
D_{Y} Z & =\nabla_{Y} Z+h(Y, Z) X \\
D_{Y} X & =-S(Y)
\end{aligned}
$$

where $Y, Z$ are tangent vectors to $H_{k}, \nabla$ is a connection on $T\left(H_{k}\right)$, the shape operator $S$ is an endomorphism of $T\left(H_{k}\right)$, and the centroaffine second fundamental form $h$ is a symmetric $(0,2)$ tensor on $H_{k}$. Note that in general, there is another term $\tau(Y) X$ in (13), where $\tau$ is a one-form on $H_{k}$. Since the position vector $X$ gives an equiaffine splitting, the $\tau$ term vanishes. See [11] for details.

Now consider

$$
\begin{aligned}
0 & =Y(d \log \sigma, Z) \\
& =\left(d \log \sigma, D_{Y} Z\right)+\left(D_{Y} d \log \sigma, Z\right) \\
& =-p h(Y, Z)+\frac{1}{c} g(Y, Z)
\end{aligned}
$$

by (7), (11) and (12). Therefore, $g(Y, Z)=\operatorname{cph}(Y, Z)$ for $Y, Z$ tangent to $H_{k}$.

\section{A remark about manifolds}

All of this theory is invariant under linear automorphisms of the cone, and therefore patches together on manifolds whose transition functions are such linear maps, in other words affine flat manifolds which admit a radiant vector field $X$. (A vector field $X$ on an affine flat manifold is radiant if $D_{Y} X=Y$ for all 
vector fields $Y$, and $D$ is the flat connection given by the affine flat structure.) It is clear that our position vector $X$ is radiant then. See e.g. [7] for details about such manifolds. See also Cheng-Yau [4] for canonical metrics on these manifolds.

Good examples of these manifolds can be constructed from certain $\mathbb{R} \mathbb{P}^{n}$ manifolds, i.e. manifolds $M$ whose coordinate charts are open sets in $\mathbb{R P}^{n}$ patched together by transition maps in $\mathbf{P G L}(n+1)$. If such a manifold admits an oriented tautological bundle (see $[9,10]$ ), then the sections of this bundle are just radial graphs over the projective coordinate charts. For a choice of inhomogeneous projective coordinates $\left[t^{1}, \cdots, t^{n}, 1\right]$, the radial graph of a function $\chi(t)$, is given by $\chi(t)\left(t^{1}, \cdots, t^{n}, 1\right) \in \mathbb{R}^{n+1}$. It is convenient to work in terms of $u$ a negative section of the dual of the tautological bundle. Then, for the hypersurface given by the radial graph of $-\frac{1}{u}$, an easy calculation in [10] shows that the centroaffine second fundamental form $h$ defined in (12) is given by

$$
h\left(\frac{\partial}{\partial t^{i}}, \frac{\partial}{\partial t^{j}}\right)=-\frac{1}{u} \frac{\partial^{2} u}{\partial t^{i} \partial t^{j}} .
$$

The positive part of the tautological bundle over such an $\mathbb{R P}^{n}$ manifold $M$ is then naturally a radiant affine manifold, and is an $\mathbb{R}^{+}$bundle over $M$. The total space of this bundle, call it $\mathcal{C}(M)$, is then made from patching together cones over the projective coordinate charts of $M$.

In [10], I showed that if $M$ is compact and admits a convex negative section $u$ of the dual of the tautological bundle, then $M$ must be the projective quotient of a bounded convex domain in $\mathbb{R}^{n}$. We can now use the splitting theorem above and a result of Shima and Yagi [13] on complete affine Kähler manifolds to extend this to complete metrics on $M$.

Theorem 2. Let $M^{n}$ be a locally projectively flat manifold without boundary. The following statements are equivalent:

1. $M$ admits an oriented tautological bundle $\tau$ and there is a negative section $u$ of the the dual bundle $\tau^{*}$ such that $-\frac{1}{u} u_{i j}$ is a complete Riemannian metric on $M$.

2. $M^{n}$ is projectively equivalent to $\Omega / \Gamma$, where $\Omega \subset \subset \mathbb{R}^{n} \subset \mathbb{R}^{n}$, $\Omega$ is convex, and $\Gamma \subset \mathbf{P G L}(n+1)$ acts discretely and properly discontinously on $\Omega$.

Proof. Assume 1. Theorem 1 above provides a complete affine Kähler metric on $\mathcal{C}(M)$. Then by [13], the universal cover of $\mathcal{C}(M)$ must be a convex domain in $\mathbb{R}^{n+1}$, which obviously must be a cone. Project along the radiant vector field to the universal cover $\tilde{M}$ of $M$, which can be put in a single inhomogenous projective coordinate chart $\mathbb{R}^{n} \subset \mathbb{R P}^{n}$. Then the section $u$ is a negative convex function on $\tilde{M}$, and therefore $\tilde{M}$ cannot contain any line.

1. follows from 2 . by the existence, due to Cheng-Yau [3, 5], of the affine sphere metric, which is complete and invariant under projective automorphisms of $\Omega$.

Remark. In the case $M$ is compact, we can recover this theorem from earlier results on affine Kähler manifolds due to Cheng-Yau [4] and Shima [12]. We can 
then consider a compact quotient $Q$ of $\mathcal{C}(M)$ by a discrete group of homotheties,

which in turn admits an affine Kähler metric given by $\frac{\partial^{2} \log V}{\partial x^{i} \partial x^{j}}$. If $p=n+1$, then $V$ is a volume form on $Q$. Under this condition, Cheng and Yau's result implies the universal cover $\mathcal{C}(\tilde{M})$ is a convex cone. Shima's result in [12] is that the universal cover of any compact affine Kähler metric is a convex domain.

The theorem also follows for $M$ compact by results of Chau on Kähler-Ricci flow [2] and Cheng-Yau on affine spheres [5]. Form an $n+1$ dimensional complex manifold $\mathcal{T}(M)$ by patching together the tube domains over each affine coordinate chart in $\mathcal{C}(M)$. Then the complete affine Kähler metric $g_{i j}$ on $\mathcal{C}(M)$ is naturally the restriction of a complete Kähler metric on $g_{i \bar{\jmath}}$ on $\mathcal{T}(M)$. For $M$ compact, it is straightforward to check that the geometry of $\left(\mathcal{T}(M), g_{i \bar{\jmath}}\right)$ is well-behaved in the sense of [2]. Chau's result says the Ricci flow carries $g_{i \bar{\jmath}}$ to a complete Kähler-Einstein metric of negative Ricci curvature. By Theorem 3 below, this metric corresponds to an affine sphere with complete affine metric over the universal cover $\tilde{M}$. The existence of such a metric implies by [5] that $\tilde{M}$ is projectively equivalent to a convex bounded domain $\Omega \subset \mathbb{R}^{n}$. See e.g. [9].

\section{Affine Spheres}

An affine sphere (with center 0 ) is a hypersurface $H$ in $\mathbb{R}^{n+1}$ whose affine normal is a constant multiple $\lambda$ of the position vector. In the case $\lambda>0$ and $H$ is locally strictly convex, we say the affine sphere is hyperbolic. It is then given by the radial graph of a function $-\frac{1}{u}$, where $u$ satisfies (2) for some $L=L(\lambda)$. The affine normal is a transverse vector field to $H$ which is invariant under the affine special linear group. It may be computed from a given transverse vector field by the procedure outlined below.

Consider the special case that $g$ is the Cheng-Yau metric as in (4), with volume form $V$.

Theorem 3. Each hypersurface $H_{k}=\{V=k\}$ is a hyperbolic affine sphere with center the origin. Under the affine Kähler metric $g$, the vector field $X$ has constant length and is orthogonal to the hypersurface $H_{k}$. The restriction $\left.g\right|_{H_{k}}$ is equal to a constant $C_{k}$ times the affine metric along $H_{k}$. Moreover, the natural foliation $\mathcal{C}=\bigcup_{s>0} s H_{1}$ gives a metric splitting

$$
(\mathcal{C}, g)=\left(\mathbb{R}^{+}, \frac{c(n+1) d s^{2}}{s^{2}}\right) \oplus\left(H_{1},[c(n+1)]^{\frac{1}{n+2}} q\right),
$$

where $q$ is the affine metric along $H_{1}$.

Proof. Most of this theorem follows immediately from Theorem 1 above. All we have to do is to verify that $H_{k}$ is an affine sphere and therefore that the centroaffine metric is a constant multiple of the affine metric.

We apply the technique in Nomizu-Sasaki [11, p. 45] to show that $X$ is a multiple of the affine normal and therefore $H_{k}$ is an affine sphere. The technique gives a formula for constructing the affine normal $\xi$ to a hypersurface $H_{k}$ given a transverse vector field $X$. We will find a function $\phi$ and a tangent vector field 
$Z$ so that $\xi=\phi X+Z$. The technique is this: First compute $\phi$ as in (15) below. Then - for $X$ equiaffine, as in our case - we have $Z=z^{i} \frac{\partial}{\partial t^{i}}$ given by the formula

$$
z^{i}=-h^{i j} \frac{\partial \phi}{\partial t^{j}} .
$$

Here $h^{i j}$ is the inverse matrix of the second fundamental form $h_{i j}=h\left(\frac{\partial}{\partial t^{i}}, \frac{\partial}{\partial t^{j}}\right)$ as in (12).

Choose $Y_{i}$ tangent to $H_{k}$ so that $\operatorname{det}\left(Y_{1}, \ldots, Y_{n}, X\right)=1$ and consider

$$
\begin{aligned}
\phi & =\left.\left|\operatorname{det}_{1 \leq i, j \leq n}\left(h\left(Y_{i}, Y_{j}\right)\right)\right|\right|^{\frac{1}{n+2}} \\
& =\left(\frac{1}{c(n+1)}\right)^{\frac{n}{n+2}}\left|\operatorname{det}_{1 \leq i, j \leq n}\left(g\left(Y_{i}, Y_{j}\right)\right)\right|^{\frac{1}{n+2}}
\end{aligned}
$$

by Theorem 1 . Now by (9) and (10),

$$
\operatorname{det}_{1 \leq i, j \leq n}\left(g\left(Y_{i}, Y_{j}\right)\right)=[c(n+1)]^{-1} \operatorname{det}_{1 \leq i, j \leq n+1}\left(g\left(Y_{i}, Y_{j}\right)\right),
$$

where in the last determinant, we set $Y_{n+1}=X$. This last determinant is equal to $V^{2}$ (our choice of $Y_{i}$ justifies changing the frame to the standard one on $\left.T\left(\mathbb{R}^{n+1}\right)\right)$, and $V=k$ on $H_{k}$. Therefore, $\phi$ is constant on $H_{k}$, and then, by (14), the affine normal is given by

$$
\xi=\phi X=[c(n+1)]^{-\frac{n+1}{n+2}} k^{\frac{2}{n+2}} X .
$$

Moreover, the affine metric along $H_{k}$ is $\phi^{-1} h=[c(n+1)]^{-\frac{1}{n+2}} k^{-\frac{2}{n+2}} g$.

Since the metric $g$ on $\mathcal{C}$ is invariant under homotheties, the global splitting of $g$ follows from the local splitting.

Remark. This correspondence gives a conceptually easy derivation of Calabi's formula [1] of the affine sphere asymptotic to the boundary of a cone $\mathcal{C}_{1} \times \mathcal{C}_{2} \subset$ $\mathbb{R}^{n+1} \times \mathbb{R}^{m+1}$ in terms of the affine spheres asymptotic to the boundaries of the convex cones $\mathcal{C}_{1}$ and $\mathcal{C}_{2}$ respectively. For each $\mathcal{C}_{i}$, construct a positive potential function $\sigma_{i}$ constant on the affine sphere and homogenous of degree $-(n+1)$. Then the Cheng-Yau metric is given by (6). Up to scaling factors, the product of two Cheng-Yau metrics is again a Cheng-Yau metric (the same is true for Kähler-Einstein metrics with the same Kähler-Einstein constant). The level sets of the volume form of this metric, i.e. hypersurfaces $\sigma_{1} \sigma_{2}=c>0$, give affine spheres asymptotic to the boundary of $\mathcal{C}_{1} \times \mathcal{C}_{2}$.

\section{Acknowledgement}

I would like to thank S.-T. Yau for introducing me to these interesting geometric equations. 


\section{References}

[1] Eugenio Calabi, Complete affine hyperspheres. I. Symposia Mathematica, Vol. X, 19-38. Academic Press, London, 1972.

[2] Albert Chau, The Ricci flow on non-compact Kähler manifolds and Kähler-Einstein metrics, Ph.D. Thesis, Columbia University, May, 2001.

[3] Shiu-Yuen Cheng and Shing-Tung Yau, On the regularity of the Monge-Ampère equation $\operatorname{det}\left(\left(\partial^{2} u / \partial x^{i} \partial x^{j}\right)\right)=F(x, u)$, Comm. Pure Appl. Math. 30 (1977), 41-68.

[4] _ The real Monge-Ampère equation and affine flat structures, Proceedings of the 1980 Beijing Symposium on Differential Geometry and Differential Equations, Vol. 1, 2, 3 (Beijing, 1980), 339-370, Science Press, Beijing, 1982.

[5] _ Complete affine Hypersurfaces. I. The completeness of affine metrics, Comm. Pure Appl. Math. 39 (1986), 839-866.

[6] Mehdi-Reza Darvishzadeh and William M. Goldman, Deformation spaces of convex real projective structures and hyperbolic affine structures, J. Korean Math. Soc. 33 (1996), 625-639.

[7] William M. Goldman, Projective geometry on manifolds, Lecture Notes for Mathematics 748B, University of Maryland, Spring, 1988.

[8] Jean Louis Koszul, Variétés localement plates et convexité, Osaka J. Math. 2 (1965), 285-290.

[9] John Churchill Loftin, Affine spheres and convex $\mathbb{R P}^{n}$ manifolds, Amer. J. Math. 123 (2001), 255-274.

[10] , Riemannian metrics on locally projectively flat manifolds, Amer. J. Math. 124 (2002), 595-609.

[11] Katsumi Nomizu and Takeshi Sasaki, Affine differential geometry. Geometry of affine immersions, Cambridge University Press, Cambridge, 1994.

[12] Hirohiko Shima, Hessian Manifolds and Convexity, Manifolds and Lie Groups, 385-392, Progr. in Math. 14, Birkhäuser, Boston, MA, 1981.

[13] Hirohiko Shima and Katsumi Yagi, Geometry of Hessian manifolds, Differential Geom. Appl. 7 (1997), 277-290.

[14] Vinberg, E. B., The theory of homogeneous convex cones, Trudy Moskov. Mat. Obšč. 12 (1963), 303-358.

Department of Mathematics, Columbia University, New York, NY 10027, U.S.A.

E-mail address: loftin@math.columbia.edu 